\title{
Introduction et messages de bienvenue
}

Intervention de Chrystel Jouan-Flahault

(Les entreprises du médicament: Leem)

Je vous souhaite la bienvenue pour cette troisième édition des rencontres Eurobiomed sur les maladies rares. Organisées tous les deux ans, ces journées constituent d'ores et déjà un événement incontournable pour tous les acteurs concernés, qu'ils soient chercheurs académiques, partenaires industriels, associations de malades ou autorités de santé. «L'innovation et les partenariats au service des malades » est le thème pivot de cette édition 2013. L'enjeu de l'innovation est bien sûr au cœur de toutes nos préoccupations. C'est ensemble que nous devons trouver les moyens de relever ces défis. Pour y parvenir, la mise en place de partenariats privé-privé ou public-privé est indispensable. C'est dans cette dynamique collégiale et partenariale que s'inscrivent ces rencontres.

Le programme de ces deux journées est construit autour de conférences plénières, de tables rondes, de sessions de speed-networking, de présentations d'entreprises et de présentations de posters.

Je remercie le pôle de compétitivité Eurobiomed, le comité scientifique et le comité d'organisation de leur implication dans la mise en œuvre de ces journées. Au nom des industries de santé, je souhaite à cette troisième édition le plus vif succès.

\section{Intervention d'Odile Kremp (Orphanet)}

Je m'associe à ces remerciements. Parmi les acteurs concernés par les maladies rares, il convient aussi de mentionner les médecins hospitaliers et les équipes soignantes. Ces deux journées réuniront 350 personnes qui auront la possibilité d'échanger et de discuter de leurs projets dans le cadre des sessions de speed-networking.

\section{Intervention de Philippe Domy (CHU de Montpellier)}

J'ai grand plaisir à me retrouver auprès d'Eurobiomed, un pôle de compétitivité compétent qui joue pleinement son rôle. Aujourd'hui et demain, nous parlerons de maladies rares. Ces dernières ne sont en fait pas si rares puisqu'elles sont 6000 à 8000 à affecter 3 millions de Français et plus de 30 millions d'Européens.
Les personnels hospitaliers sont les premiers au contact de la détresse exprimée par les malades. Pendant longtemps, ils ont été démunis face à ces maladies. Ces deux journées seront l'occasion de réunir des professionnels (hospitaliers, soignants, industriels, chercheurs, innovants, politiques et institutionnels) qui avaient rarement l'occasion de se retrouver et de parler ensemble. Ces rencontres sont la manifestation d'une volonté au service de l'intérêt général. Les hospitaliers jouent pleinement leur rôle en travaillant avec vous, parmi vous et de concert avec vous.

Le CHU de Montpellier compte 7 centres de référence maladies rares et 25 centres de compétences. II présente un fort potentiel de recherche, autour de deux grands axes que sont les maladies chroniques et les maladies rares. Le CHU gère environ 1200 protocoles de recherche actifs, et réalise 132 essais cliniques, incluant 5000 patients. II publie en moyenne 1200 articles par an. Cette année, le CHU est au $6^{\mathrm{e}}$ rang pour le score de la recherche. Notre objectif de l'année prochaine est de figurer parmi les cinq premiers.

Nous sommes heureux d'être partenaires d'un pôle de compétitivité efficace, qui consacre pour la troisième fois ces journées à la thématique des maladies rares. Vous pouvez compter sur le CHU de Montpellier, à égalité de droits et de devoirs avec les six universités, les trois autres CHU de l'interrégion. Cet ensemble réunit au sein d'Eurobiomed quelque 9000 chercheurs. Ce pôle joue un vrai rôle d'incubation et de promotion de l'innovation.

Le CHU de Montpellier souhaite intervenir à trois niveaux (la recherche fondamentale, la recherche translationnelle (de l'in vivo à la mise en place de la pratique innovante) et la recherche clinique (dans toutes ses dimensions). Toutes les forces vives du CHU sont mobilisées pour œuvrer en ce sens. Dans notre projet d'établissement et notre projet médical, le triptyque soin-enseignement-recherche est appréhendé dans sa globalité. C'est pourquoi nous affichons fièrement notre préoccupation sur la prise en soin des maladies rares.

Je vous souhaite d'excellents travaux. Ensemble, sachons promouvoir l'innovation au meilleur service des intérêts des patients qui nous font confiance.

\section{Odile Kremp}

Cette année, nous innovons puisque vous pouvez poser vos questions par SMS. Ce système vous permet de communiquer vos questions au fur et à mesure des interventions.

\section{LIENS D'INTÉR}

Les auteurs déclarent n'avoir aucun lien d'intérêt concernant les données publiées dans cet article. 